\section{WORKING FROM THE INSIDE OUT: A CASE STUDY OF MACKAY SAFE COMMUNITY}

${ }^{1}$ Dale Hanson, ${ }^{2}$ Colleen Gunning, ${ }^{3} J u d y$ Rose, ${ }^{1}$ Kathryn McFarlane, 'Richard Franklin. ${ }^{1}$ James Cook University, Australia; ${ }^{2}$ Central Queensland University, Australia; ${ }^{3}$ Mackay Hospital and Health Service, Australia

10.1136/injuryprev-2016-042156.322

Background Mackay Safe Community (MSC) was established in 2000 in response to high injury rates in the region. A community-based intervention using the International Safe Communities (ISC) model was considered strategic.

The ISC program advocates a systematic, all injury, all age group, all situation, community-based approach to injury prevention and safety promotion. MSC assumed an ecological perspective, incorporating targeted safety promotion campaigns reinforced by supportive environments and policy. By involving the community in finding its own solutions, MSC attempted to catalyse structural, social and political changes that empowered the community and ultimately, individuals within the community, to modify their environment and their behaviour to reduce the risk of injury.

Method This study used Social Network Analysis to analyse the social resources mobilised by the network. Using a snowballing methodology, the chain of relationships that constitute Mackay Safe Communities and its support network was elucidated.

Results A community network consisting of 118 members and an external support network of 50 members was established. A social network analysis conducted in 2004 indicated that the network doubled its cohesiveness while simultaneously doubling the bridging and linking relationships necessary to mobilise the resources required to implement its safety promotion agenda. A $12 \%$ reduction in Emergency Department injury presentations to Mackay Base Hospital was observed over the four-year period from 2000 to 2004. Mackay Safe Community became the $81^{\text {st }}$ International Safe Community on the $31^{\text {st }}$ August 2004.

Conclusions MSC can only be understood in its ecological context. While it was rich in social resources, human and financial resources were largely controlled by external agencies. The productivity of MSC was vulnerable to the changing policy priorities of external sponsoring agents and critically dependent on the advocacy skills of its leaders.

\section{SAFETY PROMOTION AND INJURY PREVENTION LANDSCAPE FOR CHILDREN AND THEIR FAMILIES - SAFE COMMUNITIES IN AUSTRALIA}

Barbara Minuzzo. Co-Chair Australian Safe Communities Foundation (ASCF) and Victorian Safe Communities Network (VSCN) Secretariat

\subsection{6/injuryprev-2016-042156.323}

Background Australia has national (Australian Injury Prevention Network (AIPN)) and state (Victorian Safe Communities Network (VSCN)) level networks working together. Each network was established as an independent membership based incorporated association. (The two networks gave community safety professionals a forum for support and learning when Victoria's first two municipalities were designated as International Safe Communities in 1996). The role of these networks is to support government, business and the community to develop and promote safety strategies designed to minimise the impact of unintentional injury, crime, violence and emergency situations. More recently, the Australian Safe Communities Foundation (ASCF) was established.

Methods The networks cover the nation - all attempts are made to ensure representation from each state and territory occurs at election time for both the Australian Safe Communities Foundation board and the Australian Injury Prevention Network. An eight member elected Executive Committee supported by a Secretariat manages the Victorian Safe Communities Network. Ideally there is representation from the diverse sectors working in community safety including practitioners, researchers, and policymakers.

Dissemination of information from all three organisations to its members has evolved over time from a newsletter and online listserver for members - to a Blog, Twitter, and E-Bulletin news. This approach recognises that the networks need to change how they operate to respond to the needs of the community.

Activities undertaken have also evolved over time from consultations/support/certification as a safe community, face-to-face workshops/seminars, and a yearly conference to a conference every two years, online webinars, some face to face seminars, consultations with its members and writing position papers to influence Government.

Sustainability of the three organisations and the success of safe communities already long established was borne of the strong commitment by individuals and a shift in culture, and policy at key stakeholder and local government and organisational level. This culture shift encourages partnerships, networks and relationship building across sectors for the betterment of Safe Communities, by directing or participating in working groups. This provides a platform to progress safety issues locally as well as through government decision making processes. A healthy financially sustainable plan for the three networks continues to be an ongoing challenge depending on the national and state government agenda at the time

Results These networks facilitate a collaboration, to provide information and expertise on a range of community safety issues - across government and non-government agencies which influence policy development, support knowledge sharing and development and dissemination of resources.

These networks contribute to the expansion of safer communities, providing a platform for researchers and injury prevention practitioners to work with communities, implementing a broad multi-disciplinary approach to provide the best advice for individual community need. Tangible examples of the benefits of these networks include:

- Connecting communities seeking formal accreditation with other communities who have experience of the processes involved.

- Providing opportunities for networking and professional development through seminars and conferences conducted in partnership with other agencies and groups.

Conclusions Success comes to Australian local governments working to gain international safe community's accreditation The activities focus on promoting evidence based best practice and strength based approaches to early intervention. 